\title{
Enzyme-Powered Liquid Metal Nanobots Endowed with Multiple Biomedical Functionalities
}

Dandan Xu, ${ }^{a, b, t}$ Jing Hu, ${ }^{c, t}$ Xi Pan, ${ }^{a, b}$ Samuel Sánchez, ${ }^{d, e, f}$ Xiaohui Yan, , * and Xing $M a^{a, b, *}$

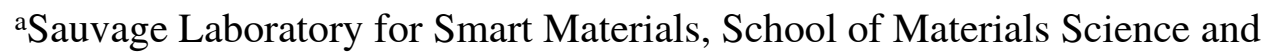
Engineering, Harbin Institute of Technology (Shenzhen), Shenzhen 518055, China

bShenzhen Bay Laboratory, No. 9 Duxue Road, Shenzhen 518055, China

'State Key Laboratory of Molecular Vaccinology and Molecular Diagnostics, Center for Molecular Imaging and Translational Medicine, School of Public Health, Xiamen University, Xiamen, 361005, China

${ }^{\mathrm{d} S c h o o l ~ o f ~ M a t e r i a l s ~ S c i e n c e ~ a n d ~ E n g i n e e r i n g, ~ H a r b i n ~ I n s t i t u t e ~ o f ~ T e c h n o l o g y ~}$ (Shenzhen), Shenzhen 518055, China

eInstitució Catalana de Recerca i Estudis Avançats (ICREA), Pg. Lluís Companys 23, Barcelona 08010, Spain

fInstitute for Bioengineering of Catalonia (IBEC), The Barcelona Institute of Science and Technology, Baldiri Reixac 10-12, Barcelona 08028, Spain 


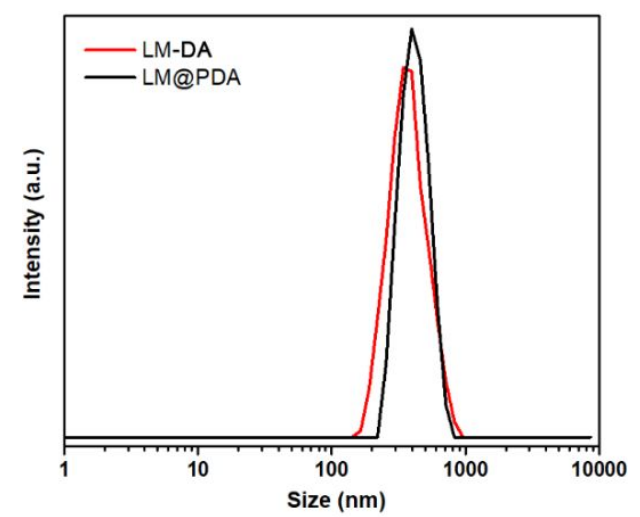

Figure S1. Size distributions of LM-DA and LM@PDA.
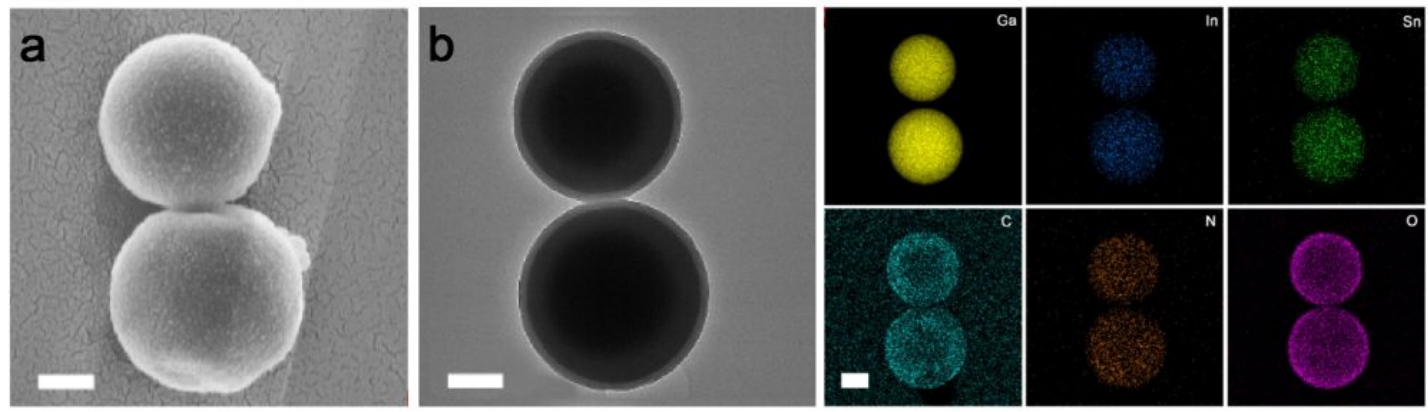

Figure S2. a) SEM image and b) TEM image with corresponding elemental mapping by EDX of LM@PDA. Scale bar=100 nm.
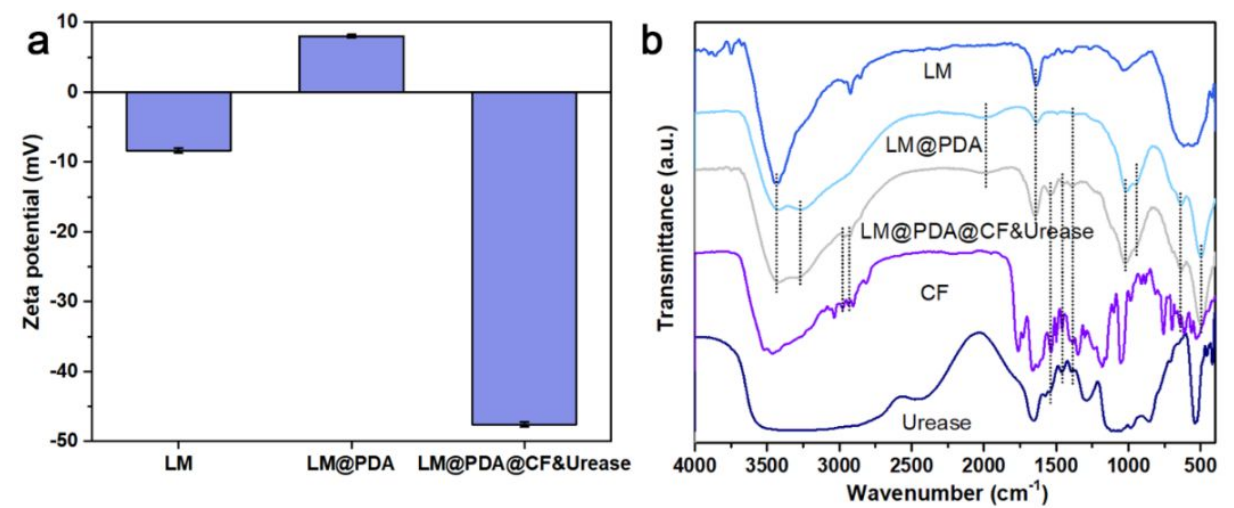

Figure S3. a) Zeta potentials of LM, LM@PDA and LPCU. b) FT-IR spectra of LM, LM@PDA, LPCU, CF and Urease. Scale bar=100 nm.

\section{Discussion:}

Figure S3a: The zeta potential value of nanosized LM obtained by sonication without any modification is negative, while that of LM@PDA is positive suggesting successfully coating of PDA. Then, LPCU has negative zeta potential, suggesting the further loading of CF and urease on the external surface. 
Figure S3b: The spectra of LM@PDA and LPCU both possess bands centered at 1637 and $3415 \mathrm{~cm}^{-1}$ arising from the LM, and bands at 499, 1390, 1992 and $3260 \mathrm{~cm}^{-1}$ from the PDA, among which the band at $1,390 \mathrm{~cm}^{-1}$ also from the CF and the urease. ${ }^{1-2}$ Meanwhile, the spectra of LPCU show the bands at 636, 2978 and $2937 \mathrm{~cm}^{-1}$ arising from the $\mathrm{CF}$ and the bands at 1543 and $1456 \mathrm{~cm}^{-1}$ arousing from both the $\mathrm{CF}$ and the urease. In addition, the peak at $957 \mathrm{~cm}^{-1}$ can be assigned to $\mathrm{Ga}-\mathrm{OH}$ bond ${ }^{3}$ and that at $1,261 \mathrm{~cm}^{-1}$ is due to the stretching band of $\mathrm{C}-\mathrm{OH}$ from carboxyl or phenolic $\mathrm{OH},{ }^{4}$ which indicates that PDA assisted the dispersion of LM nanodroplets in aqueous solution by chemical bonding. In addition, the spectra of LPCU show the stretching band of NH group at $636 \mathrm{~cm}^{-1}, \mathrm{C}-\mathrm{H}$ band at $2978 \mathrm{~cm}^{-1}$ and $\mathrm{OH}$ band at $2937 \mathrm{~cm}^{-1}$, which are attributed to the presence of CF. The band at $1543 \mathrm{~cm}^{-1}$ from the bending of $\mathrm{NH}$ and the stretching of $\mathrm{C}-\mathrm{N}$, and that at $1456 \mathrm{~cm}^{-1}$ from the bending of $\mathrm{CH}$ and the vibrating of $\mathrm{C}=\mathrm{C}$ skeleton appear in the spectrum of $\mathrm{LPCU}$, which are attributed to the presence of CF and urease.
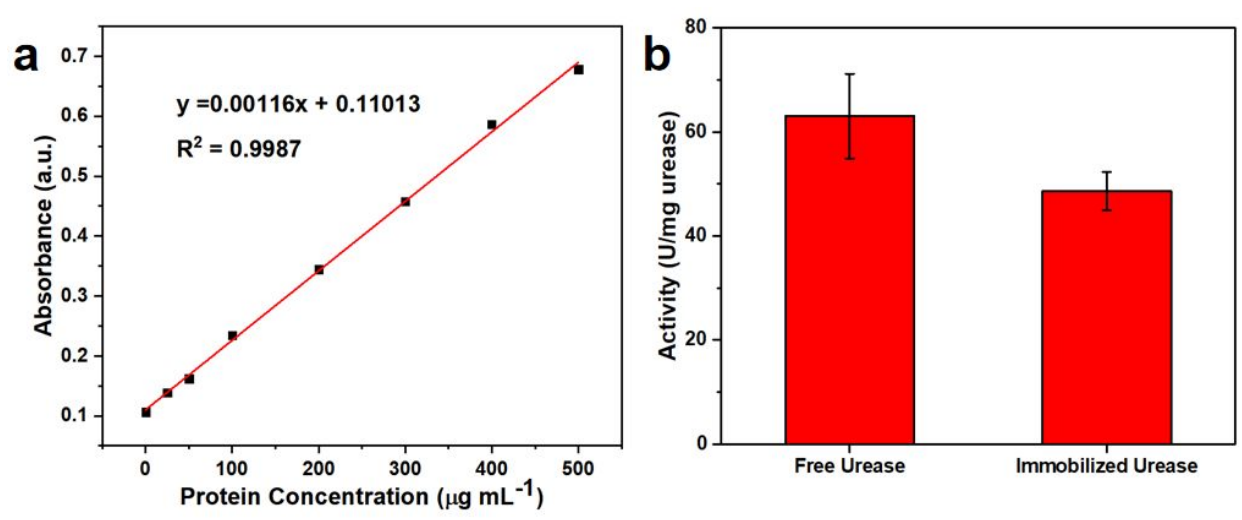

Figure S4. a) The plot showing the dependence of the absorbance of BCA protein standard solution on its concentration, b) Activity of free urease and immobilized urease onto the nanobot. 


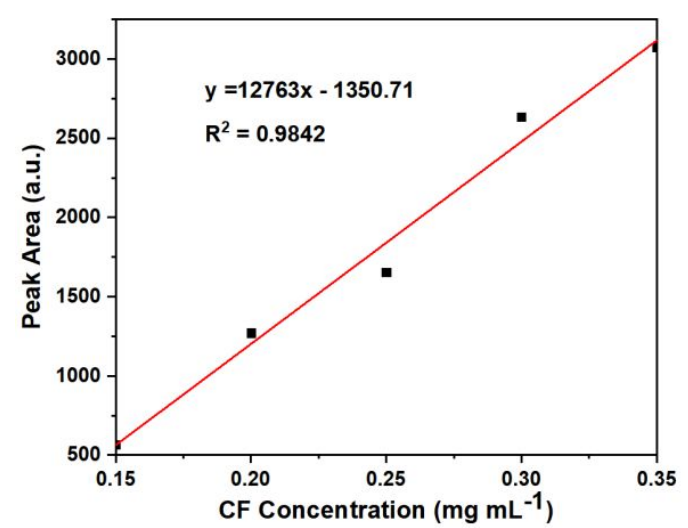

Figure S5. Variation of the peak area of CF standard solution with different concentrations.

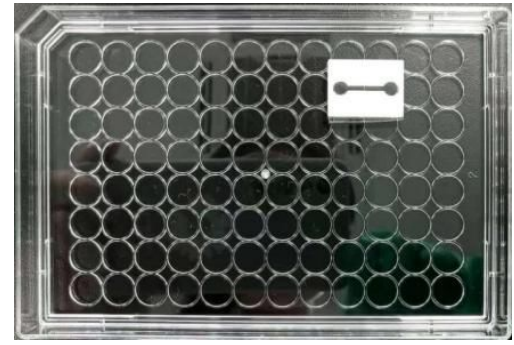

Figure S6. Photo of the experiment setup used in the section of Evaluation of Chemotactic Movement of the nanobots.

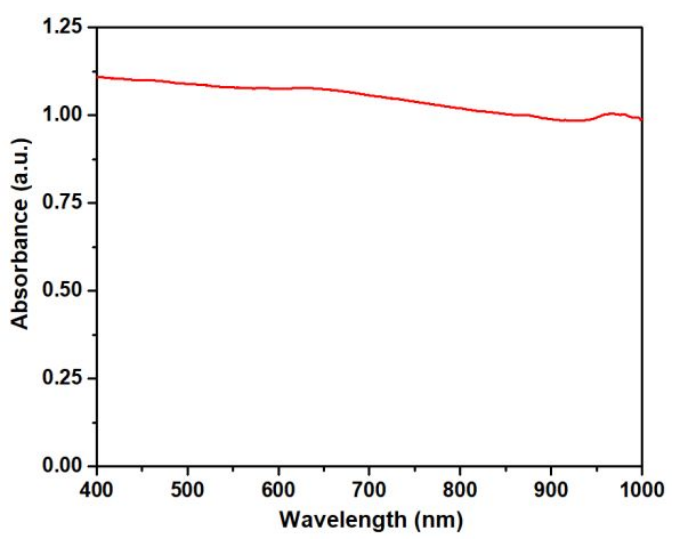

Figure S7. UV-vis spectrum of LPCU $\left(1 \mathrm{mg} \mathrm{mL}^{-1}\right)$. 


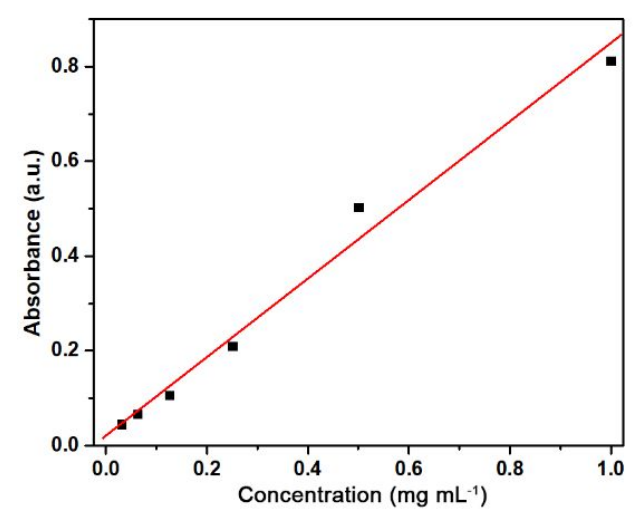

Figure S8. Variation of the UV-vis absorbance of LPCU with different concentrations at $700 \mathrm{~nm}$.

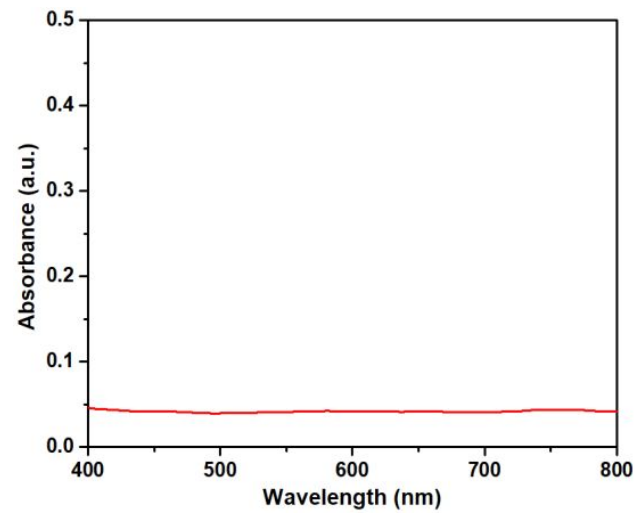

Figure S9. UV-vis spectrum of urea solution $(25 \mathrm{mM})$.
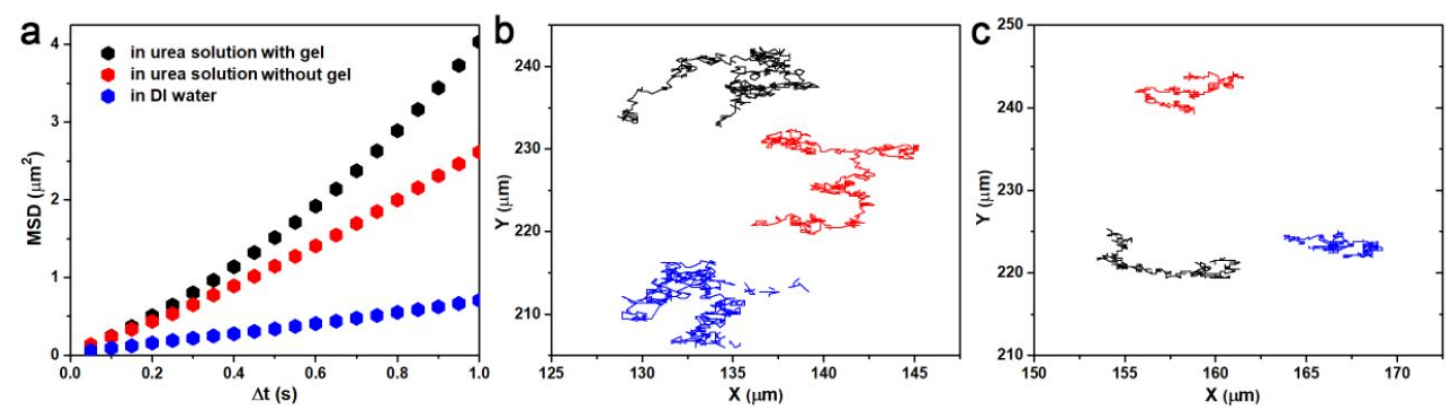

Figure S10. (a) Plots of mean-square-displacement (MSD) versus time interval $(\Delta t)$ of LPCU swimming in urea solution with gel, in urea solution without gel and in DI water $(\mathrm{N}=15)$. Representative tracking trajectories of LPCU swimming (b) in urea solution without gel and (c) in DI water within $30 \mathrm{~s}$. 


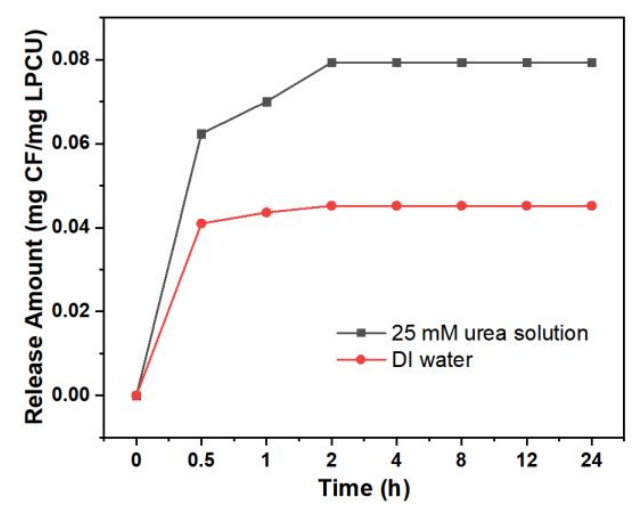

Figure S11 Release curves of CF from LPCU in $25 \mathrm{mM}$ urea solution and DI water within 24 h, respectively.

Discussion:

Figure S11: The release amount of CF was continuously increasing within $2 \mathrm{~h}$, after which the amount of CF basically remained unchanged. The cumulative amount of CF that released from LPCU nanobots incubated in deionized water and urea solution for $24 \mathrm{~h}$ were up to 0.0452 and $0.0793 \mathrm{mg} \mathrm{mL}^{-1}$, respectively, which were about $11.08 \%$ and $19.44 \%$ of the loading amount of CF.

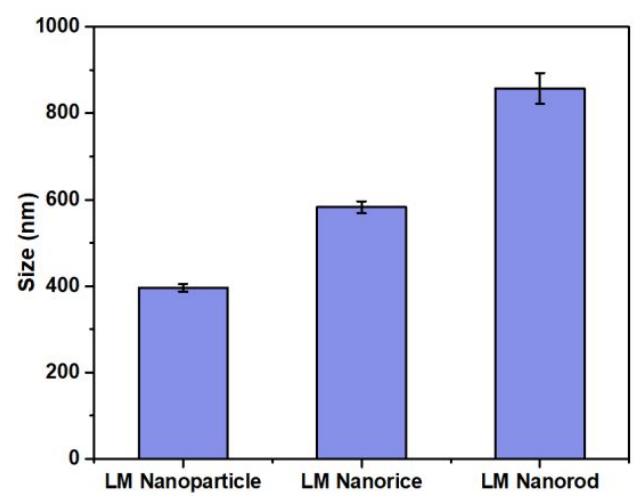

Figure S12. Size distributions of LM nanoparticle, LM nanorice and LM nanorod.
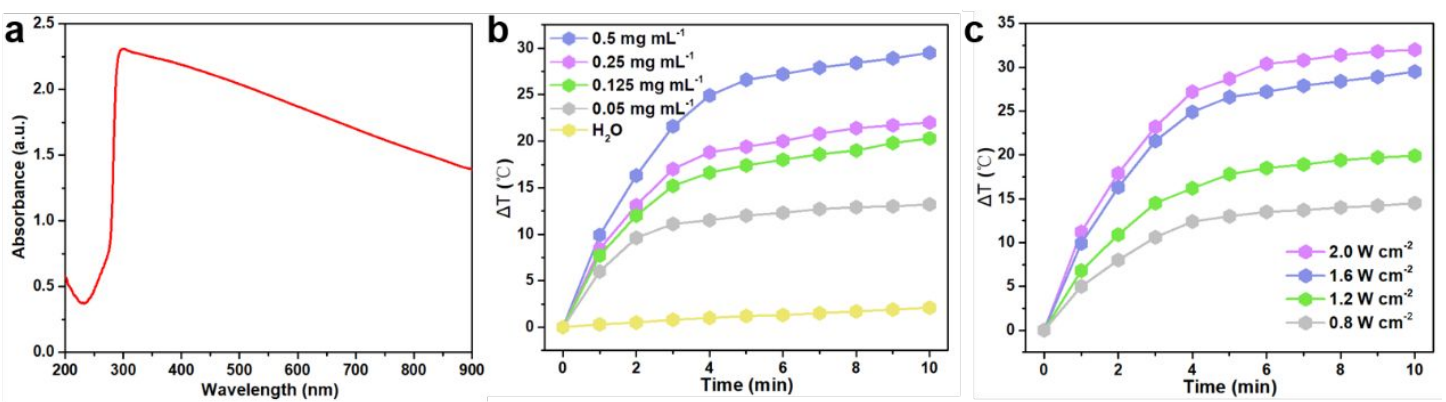

Figure S13. (a) Absorbance of LM nanorices $\left(1 \mathrm{mg} \mathrm{mL}^{-1}\right)$, photothermal responses of LM nanorices with various (b) power intensities and (c) concentrations under NIR laser 
irradiation (808 nm, $10 \mathrm{~min}) . \mathrm{H}_{2} \mathrm{O}$ was used as control.

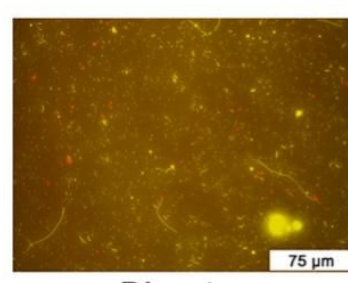

DI water

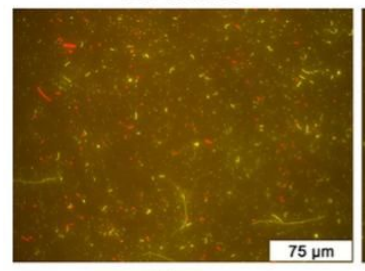

Urea

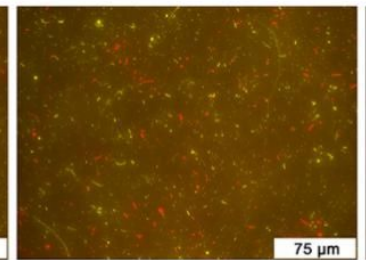

LM@PDA

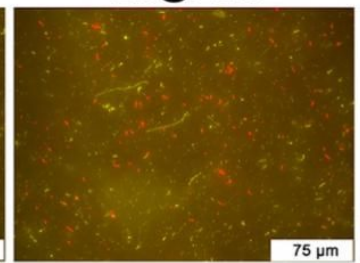

LM@PDA + Urea

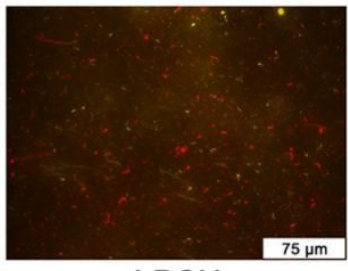

LPCU

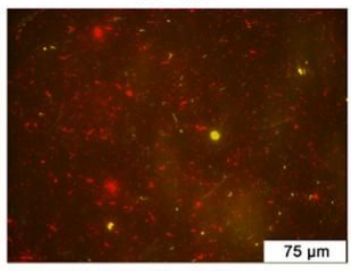

LPCU + Urea

Figure S14. Merged images of the dead E. coli (red) and the live E. coli (yellow) after different treatments, respectively.

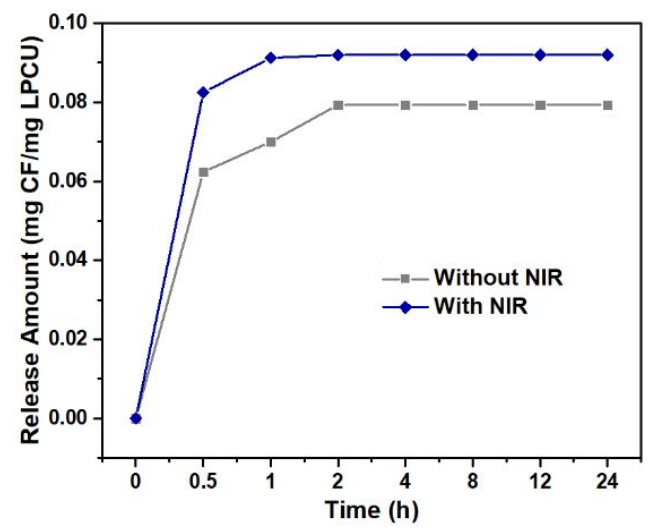

Figure S15 Release curves of CF from LPCU in $25 \mathrm{mM}$ urea solution with/without NIR light irradiation for 15 min twice, respectively.

\section{Discussion:}

Figure S15: The release amount of CF obviously increased within the first hour after NIR light treatment and then basically remained unchanged. The cumulative amount of CF that released from LPCU nanobots with and without light treatment were up to 0.0920 and $0.0793 \mathrm{mg} \mathrm{mL}^{-1}$, respectively, which were about $22.55 \%$ and $19.44 \%$ of the loading amount of CF. Reasons for such increasing release are speculated as follows. On the one hand, the photothermal effect produced by the LPCU nanobots under NIR light irradiation would accelerate drug release by probably breaking the surface molecule bonding. On the other hand, after the transformation process, the PDA would partially degrade and the released small PDA fragments performing Brownian motion might also help the release of drug molecules. 


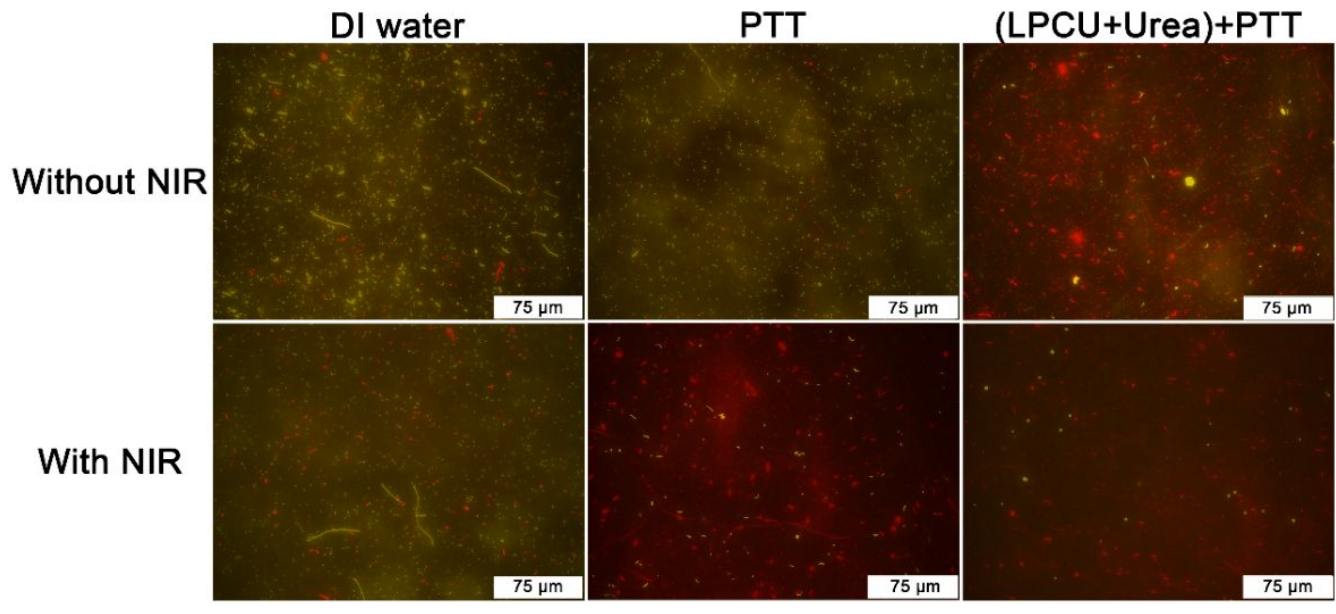

Figure S16. Merged images of the dead E. coli (red) and the live E. coli (yellow) after different treatments, respectively.

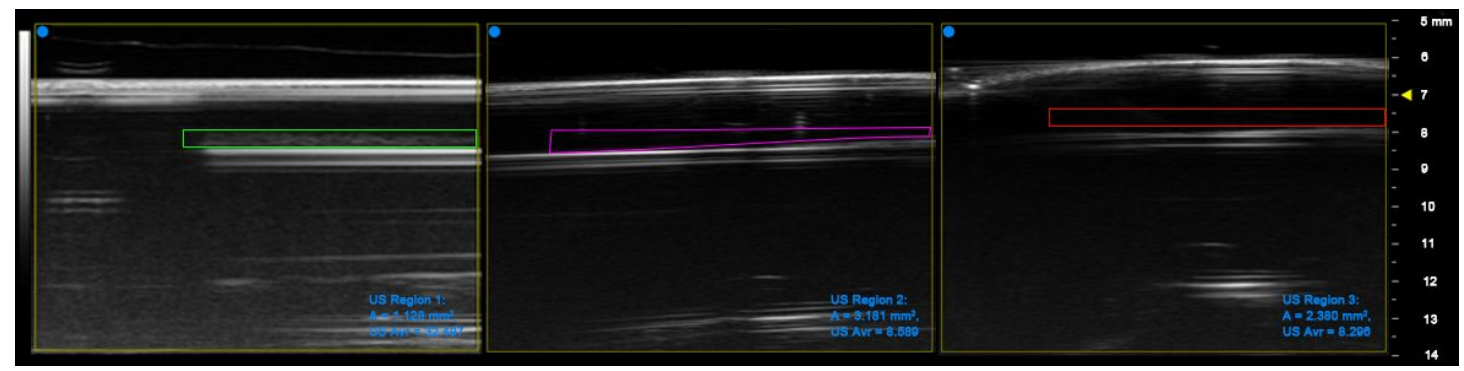

Figure S17. US images of LPCU nanobots $\left(10 \mathrm{mg} \mathrm{mL}^{-1}\right)$ inside a microfluidic vessel where the velocity of fluid varies from 10 to 2 to $1 \mu \mathrm{L} \mathrm{s}^{-1}$ (from left to right).

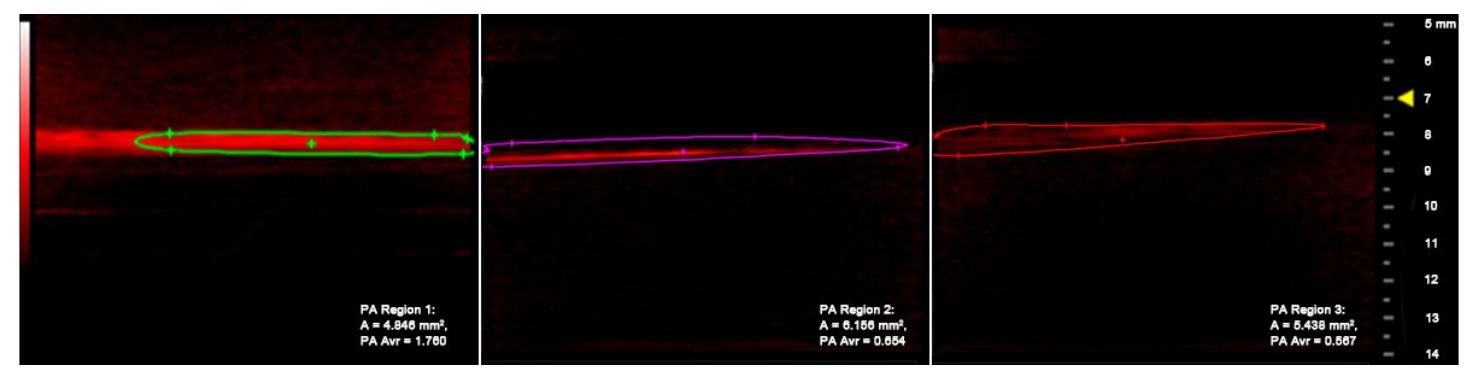

Figure S18. PA images of LPCU nanobots $\left(10 \mathrm{mg} \mathrm{mL}^{-1}\right)$ inside a microfluidic vessel where the velocity of fluid varies from 10 to 2 to $1 \mu \mathrm{L} \mathrm{s}^{-1}$ (from left to right). 


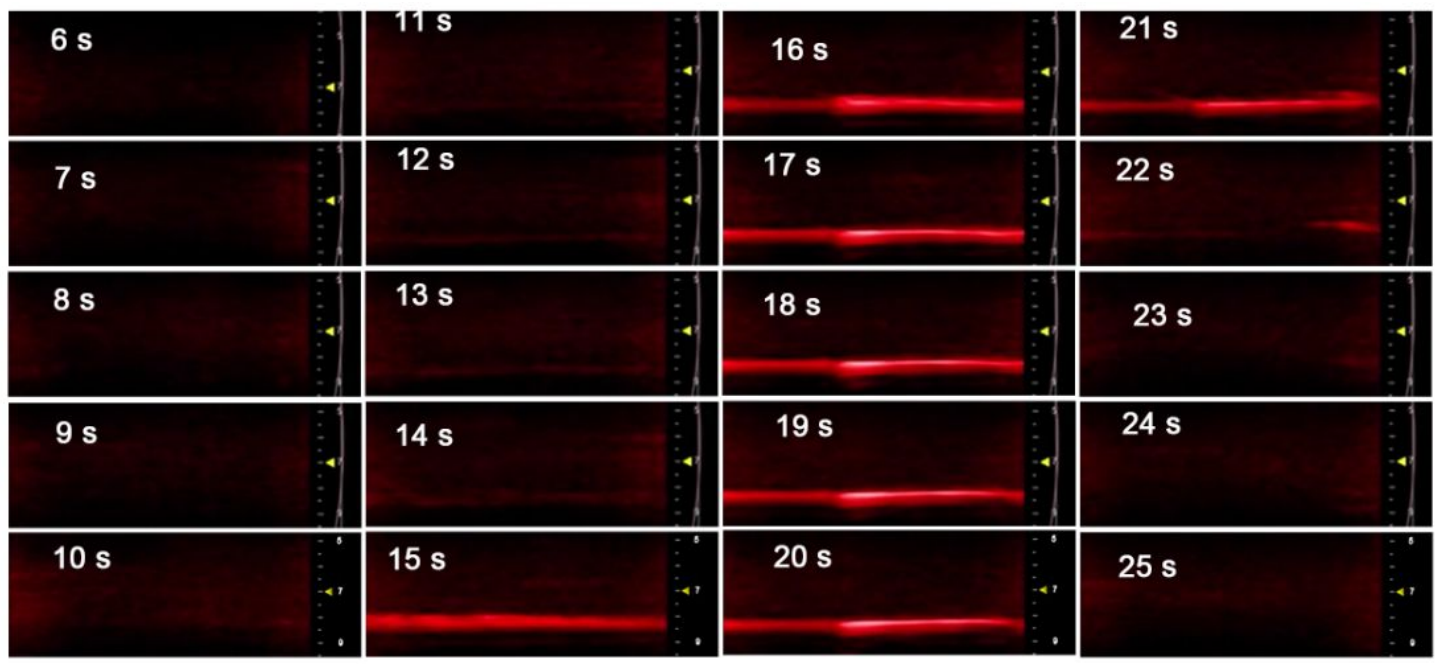

Figure S19. Time-lapse PA images of LPCU nanobots $\left(10 \mathrm{mg} \mathrm{mL}^{-1}\right)$ inside a microfluidic vessel $(\mathrm{d}=2 \mathrm{~mm})$ where the velocity of fluid is $10 \mu \mathrm{L} \mathrm{s}^{-1}$

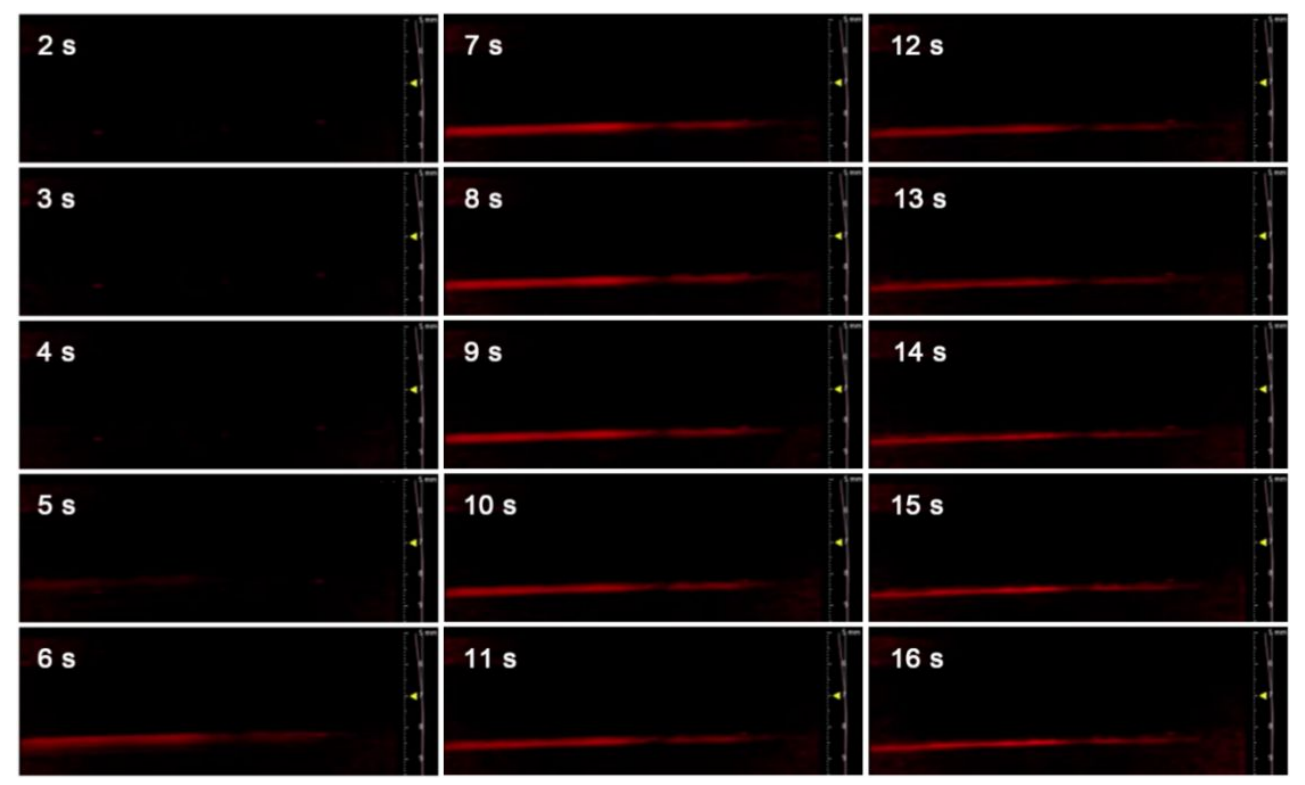

Figure S20. Time-lapse PA images of LPCU nanobots $\left(10 \mathrm{mg} \mathrm{mL}^{-1}\right)$ inside a microfluidic vessel $(\mathrm{d}=2 \mathrm{~mm})$ where the velocity of fluid is $2 \mu \mathrm{L} \mathrm{s}^{-1}$ 

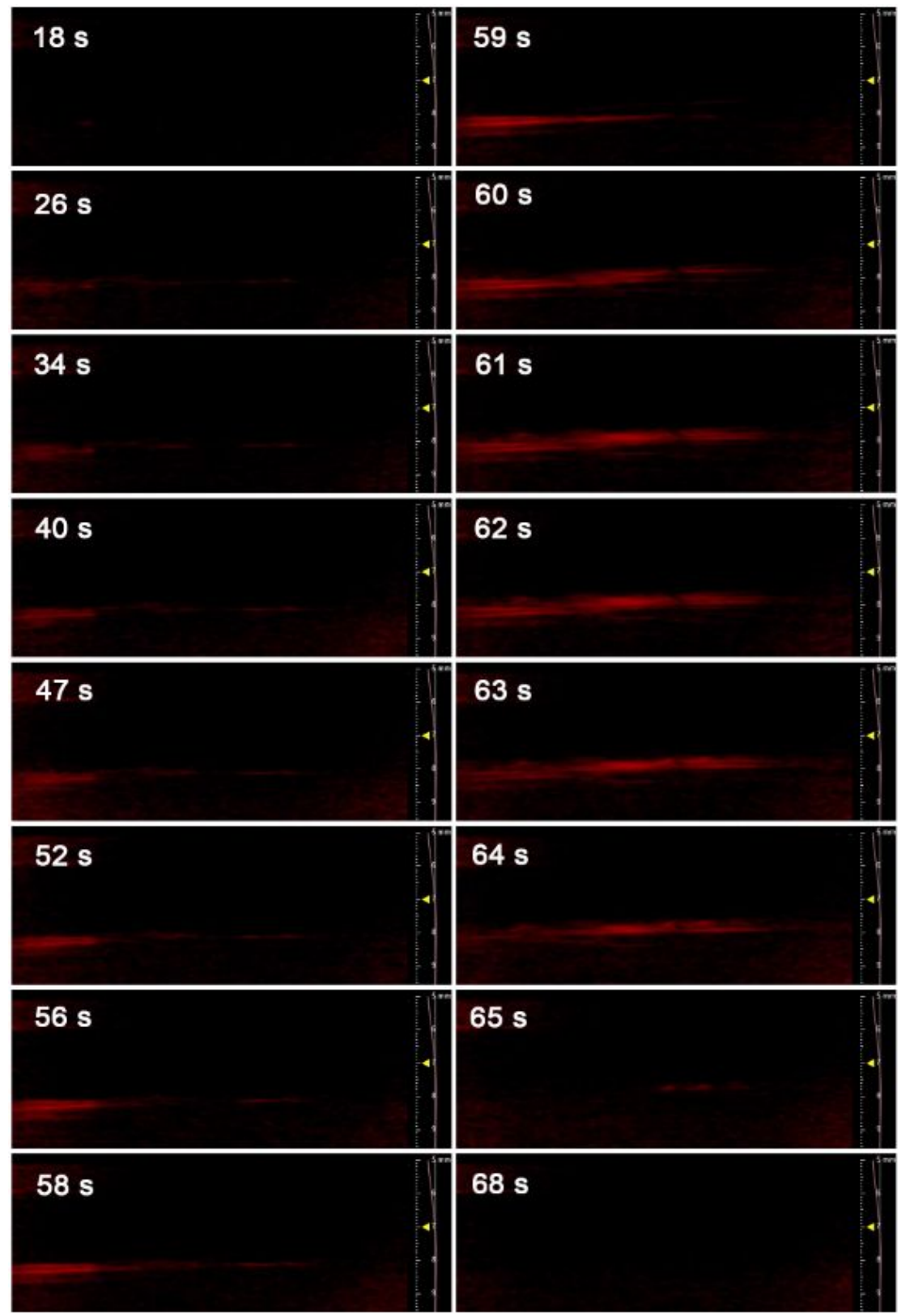

Figure S21. Time-lapse PA images of LPCU nanobots $\left(10 \mathrm{mg} \mathrm{mL}^{-1}\right)$ inside a microfluidic vessel $(\mathrm{d}=2 \mathrm{~mm})$ where the velocity of fluid is $1 \mu \mathrm{L} \mathrm{s}^{-1}$ 


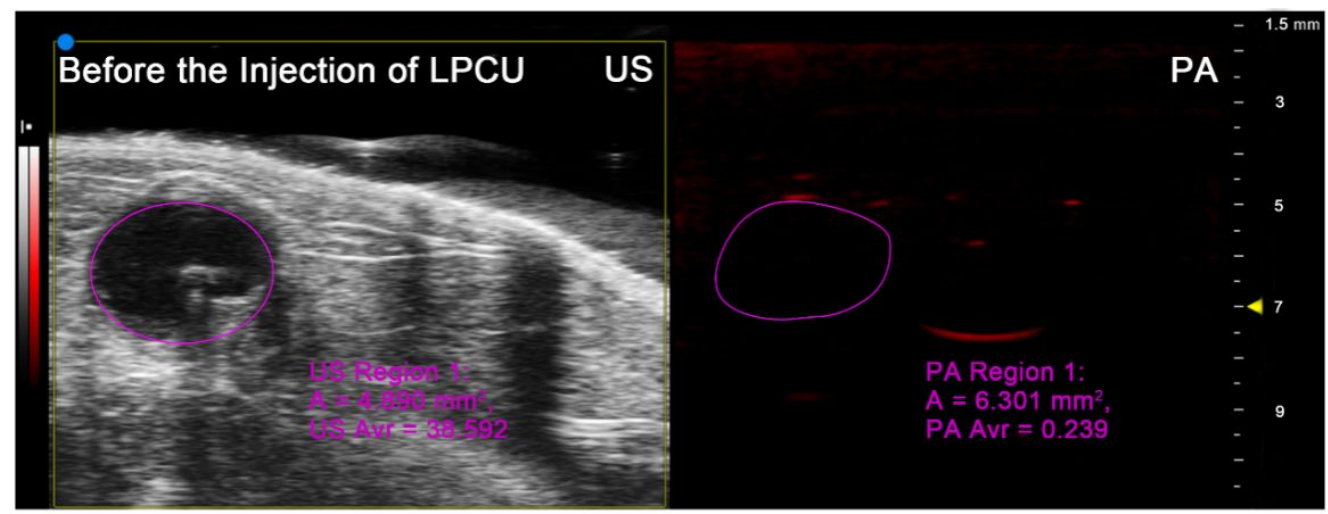

Figure S22. US and PA images of the bladder of the mouse before the injection of the nanobots $\left(10 \mathrm{mg} \mathrm{mL}^{-1}\right)$.

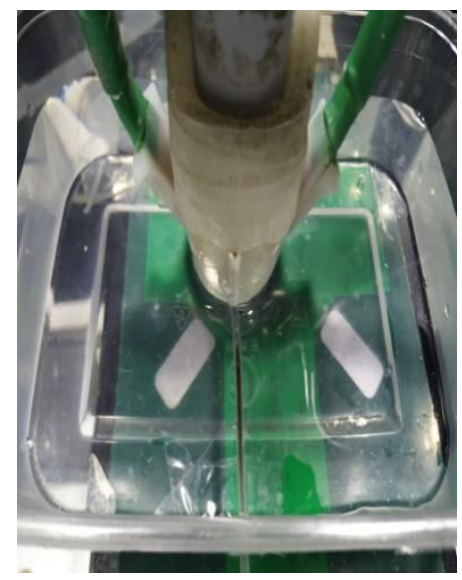

Figure S23. A microfluidic vessel model, with an US-PA detector on its top, suspended in the box that filled with water for dynamic tracking of moving LPCU.

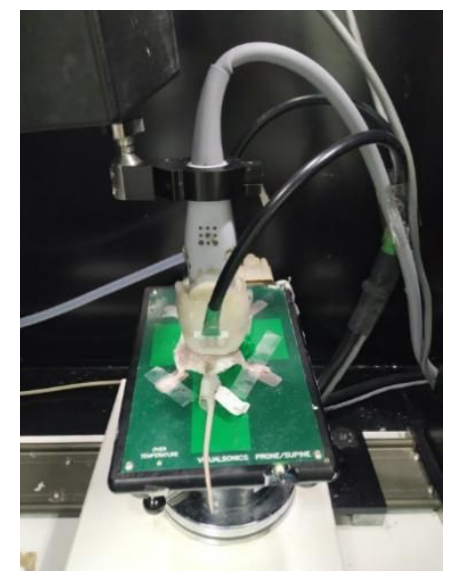

Figure S24. An US-PA detector on top of the bladder of a mouse after the injection of LPCU for dynamic imaging. 


\section{REFERENCES}

(1) Mbonyiryivuze, A.; Mwakikunga, B.; Dhlamini, S. M.; Maaza, M., Fourier Transform Infrared Spectroscopy for Sepia Melanin. Phys. Mater. Chem. 2015, 3, 25 29.

(2) Zangmeister, R. A.; Morris, T. A.; Tarlov, M. J., Characterization of Polydopamine Thin Films Deposited at Short Times by Autoxidation of Dopamine. Langmuir 2013, 29, 8619-8628.

(3) Taş, A. C.; Majewski, P. J.; Aldinger, F., Synthesis of Gallium Oxide Hydroxide Crystals in Aqueous Solutions with or without Urea and Their Aalcination Behavior. J. Am. Ceram. Soc. 2002, 85, 1421-1429.

(4) Bronze-Uhle, E. S.; Paulin, J. V.; Piacenti-Silva, M.; Battocchio, C.; Rocco, M. L. M.; Graeff, C. F. d. O., Melanin Synthesis under Oxygen Pressure. Polym. Int. 2016, 65, 1339-1346. 